\title{
Lithium associated Hyperparathyroidism (LAH): Cinacalcet is an effective alternative treatment option
}

\author{
Jampana N1, Rajkanna J2, Oyibo SO2', Sagi SV² \\ ${ }^{1}$ Department of Old age Psychiatry, Cambridgeshire \& Peterborough NHS Foundation Trust \\ ${ }^{2}$ Department of Endocrinology, Peterborough City Hospital, Peterborough, UK
}

\section{Introduction}

Lithium associated hyperparathyroidism (LAH) resulting in hypercalcemia is a known problem. Treatment options are limited in frail elderly patients with multiple co-morbidities, in whom parathyroid surgery could be dangerous. Cinacalcet, a calcimimetic agent can be an alternative option. We report a case of an elderly lady on Lithium, who was initiated on Cinacalcet for hypercalcaemia and has had to continue the Lithium for her bipolar affective disorder.

\section{Case}

A 78 year old lady was admitted with severe hypercalcaemia (serum calcium = $3.03 \mathrm{mmol} / \mathrm{L}$, serum PTH $=10.4 \mathrm{pmol} / \mathrm{L}$ ). She has a bipolar affective disorder, other medical co-morbidities and has been on long term Lithium treatment. Several years ago she had severe LAH treated with three-and-a-half-gland parathyroidectomy and has had normal serum calcium levels ever since. During this admission her lithium was stopped and hypercalcemia responded to fluids and intravenous Bisphosphanates. During outpatient monitoring her serum calcium started rising (serum calcium $=2.81 \mathrm{mmo} / \mathrm{L}$ ) over time despite stopping Lithium.

After considering risks and benefits of completion of parathyroidectomy, she was commenced on Cinacalcet $30 \mathrm{mg}$ once daily. Her calcium levels returned and settle back to the normal range (average serum calcium $=2.37 \mathrm{mmo} / \mathrm{L}$ ).

Unfortunately her bipolar affective disorder had relapsed since stopping lithium therapy. With the consent of the patient and her family, we restarted lithium while continuing her Cinacalcet. Her calcium levels were monitored closely. After a year of treatment with the combination of low-dose Cinacalcet and Lithium, her serum calcium and PTH levels have remained in the normal range (see Table 1).
Table 1 - Calcium \& Parathormone (PTH) levels while on and off Lithium (Li) \& Cinacalcet therapy

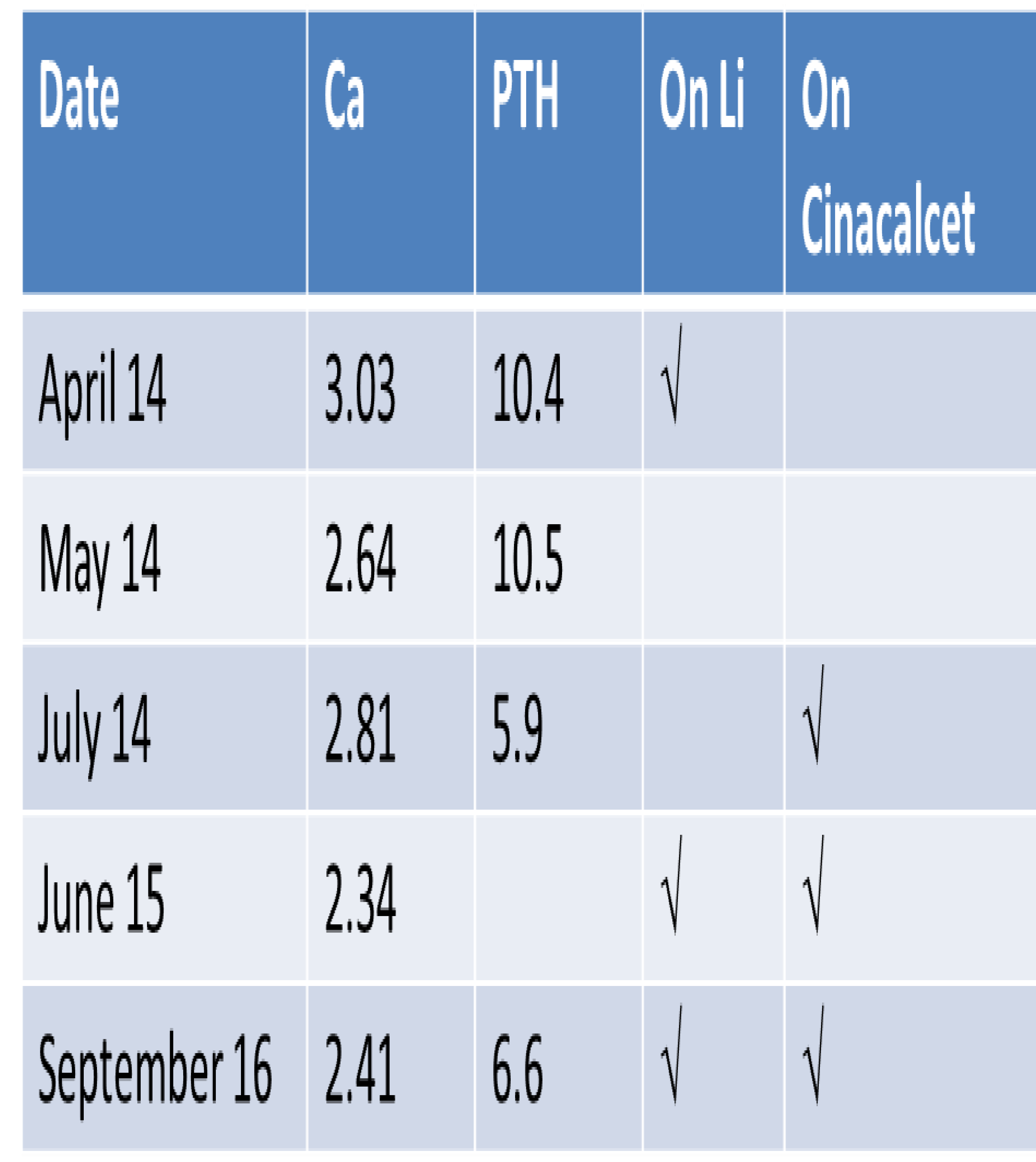

\section{Conclusions}

The management of LAH can be challenging in elderly patients with complex psychiatric and multiple medical co-morbidities. Cinacalcet appears to be an effective alternative treatment for such patients while still on Lithium, however further studies are needed for its regular use. 